\title{
Original
}

\section{Modelo CBCRisk para determinar el riesgo de cáncer de mama contralateral en el cáncer de mama esporádico}

\author{
María Domingo Bretón ${ }^{a, *}$, Marta Allué Cabañuz ${ }^{a}$, Néstor Castán Villanueva ${ }^{a}$, \\ María Dolores Arribas del Amo ${ }^{b}$, Ismael Gil Romea ${ }^{b}$ y Antonio Güemes Sánchez ${ }^{b}$ \\ a Servicio de Cirugía General, Hospital Clínico Universitario Lozano Blesa, Zaragoza, España \\ b Servicio de Cirugía General, Unidad de Mama, Hospital Clínico Universitario Lozano Blesa, Zaragoza, España
}

\section{INFORMACIÓN DEL ARTÍCULO}

\section{Historia del artículo:}

Recibido el 29 de septiembre de 2020 Aceptado el 16 de noviembre de 2020

On-line el $\mathrm{xxx}$

Palabras clave:

Cáncer de mama

Mastectomía reductora de riesgo

Mastectomía profiláctica

contralateral

Riesgo

CBCRisk

\section{R E S U M E N}

Introducción: La mayoría de los cánceres de mama (CM) se diagnostican en mujeres sin antecedentes familiares y no portadoras de mutaciones de riesgo. En las últimas décadas se ha producido un aumento de mastectomías profilácticas contralaterales (MPC) en estas pacientes. El CBCRisk es un modelo que calcula el riesgo absoluto de cáncer de mama contralateral (CMC) y pretende servir para el asesoramiento de pacientes con CM esporádico sobre la MPC.

Método: Análisis observacional retrospectivo de pacientes con un cáncer de mama esporádico sometidas a MPC durante 2017-2019. Análisis descriptivo, comparativo y de regresión logística univariante para identificar factores predictivos de LMAR y/o CMC oculto. Evaluación del modelo CBCRisk publicado en 2017 y distintos valores límite para la recomendación de MPC.

Resultados: Se seleccionaron 42 pacientes. Incidencia de LMAR y cáncer oculto (CO) menor que la descrita en la literatura (9,52\% LMAR, 2,38\% CO). Ninguna de las variables evaluadas alcanzó significación estadística para la predicción de lesiones. El valor de CBCRisk a cinco años medio en pacientes con hallazgos patológicos fue de 2,08 (DE 0,97), superior al CBCRisk medio del conjunto $(1,87 \pm 0,91)$ y del subgrupo de MPC sin hallazgos patológicos $(1,84 \pm$ $0,91)$. Solo el CBCRisk $\geq 3$ resultó significativo $(\mathrm{p}=0,04)$ para la predicción de hallazgos patológicos.

Conclusión: Las pacientes con CM esporádico deben ser adecuadamente informadas de los riesgos y beneficios estimados de la MPC. El CBCRisk puede ser útil para el asesoramiento de estas pacientes, pero precisa validación en cohortes más amplias y prospectivas.

(C) 2020 AEC. Publicado por Elsevier España, S.L.U. Todos los derechos reservados.

\footnotetext{
* Autor para correspondencia.

Correo electrónico: mdominbre@gmail.com (M. Domingo Bretón).

https://doi.org/10.1016/j.ciresp.2020.11.007

0009-739X/C) 2020 AEC. Publicado por Elsevier España, S.L.U. Todos los derechos reservados.
} 
Keywords:

Breast cancer

Risk-reducing mastectomy

Contralateral prophylactic

mastectomy

Risk assessment

CBCRisk

\section{CBCRisk model to determine the risk of contralateral breast cancer in sporadic breast cancer}

\begin{abstract}
A B S T R A C T
Introduction: The great majority of breast cancer (BC) cases are diagnosed in women who have no known family history of the disease and are not carriers of any risk mutation. During the past few decades an increase in the number of contralateral prophylactic mastectomy (CPM) has been produced in these patients. The CBCRisk model calculates the absolute risk of suffering from contralateral breast cancer ( $\mathrm{CBC}$ ); thus, it can be used to counselling patients with sporadic breast cancer.

Method: An observational, retrospective study including sporadic breast cancer patients treated with contralateral prophylactic mastectomy has been conducted between 2017 and 2019. A descriptive and comparative study with one variation of logistic regression has been carried out in order to identify predictive factors of occult tumors (OT) and medium/high risk damage (MHRD). Evaluation of the CBCRisk model published in 2017 and different limit values for the CPM recommendation.

Results: 42 patients were selected. Incidence of MHRD and OT was lower than that described in the literatura (9.52\% MHRD, $2.38 \% \mathrm{OT})$. None of the evaluated variables reached statistical significance for predicting injuries. The average value of CBCRisk 5 years ahead found in patients with pathological findings was 2.08 (SD 0.97), higher than the average value of the whole group $(1.87 \pm 0.91)$ and the subgroup without pathological findings $(1.84 \pm 0.91)$. Only values $\geq 3$ for CBCRisk were considered statistically significant $(p=0.04)$ for the prediction of histological lesions.

Conclusion: Patients with sporadic breast cancer should be adequately informed about the estimated risks and benefits of undergoing a contralateral prophylactic mastectomy. The CBCRisk may be useful for the counseling of these patients, but it requires validation in larger and prospective cohorts.
\end{abstract}

(C) 2020 AEC. Published by Elsevier España, S.L.U. All rights reserved.

\section{Introducción}

La mastectomía profiláctica contralateral (MPC) ha sido evaluada como estrategia para la reducción del riesgo de cáncer de mama contralateral (CMC) en pacientes con un cáncer de mama esporádico (es decir, sin mutaciones en los principales genes asociados al cáncer de mama hereditario, y sin una fuerte historia familiar $)^{1}$.

La MPC constituye la estrategia terapéutica que aporta mayor reducción del riesgo de CMC, disminuye la necesidad de controles, y con ello, la preocupación y ansiedad de las pacientes, además de aportar beneficios en cuanto a simetrización. Sin embargo, se trata de un procedimiento comprometido: es agresivo, irreversible, duplica el riesgo de complicaciones quirúrgicas ${ }^{2}$, puede retrasar la administración de terapias adyuvantes y con frecuencia requiere otras intervenciones (fundamentalmente por asociar algún tipo de reconstrucción mamaria). Puede asociarse con dolor crónico $^{3}$ e influir negativamente en la salud mental y sexual de las pacientes ${ }^{4}$. Además, el beneficio en términos de supervivencia de la MPC en mujeres no portadoras de mutaciones BRCA no ha sido claramente demostrado, a excepción quizá de las mujeres menores de 49 años con tumores triple negativo ${ }^{5}$.

Las indicaciones de consenso ${ }^{1}$ para la MPC incluyen el antecedente de irradiación supradiafragmática antes de los
30 años y la mutación demostrada BRCA 1/2. Puede considerarse en el caso de portadoras de mutaciones CHEK2/PTEN/ p53/PALB2/CDH1, pacientes con fuerte historia familiar sin mutaciones de riesgo demostradas, o en caso de importante asimetría tras la mastectomía unilateral (con o sin reconstrucción).

En las últimas décadas, hemos asistido a un aumento en la realización de MPC en pacientes con CM esporádico, más notable entre la población estadounidense que en la europea, por factores sociales y culturales aún no aclarados ${ }^{6,7}$. Este hecho resulta paradójico, ya que el diagnóstico precoz del CM junto con la mejoría en las terapias adyuvantes ha permitido, por un lado, aumentar las posibilidades de conservación de la mama (ampliándose las indicaciones de la cirugía conservadora) $\mathrm{y}$, por otro, disminuir la recurrencia, mortalidad e incidencia de $\mathrm{CMC}^{8}$.

Algunos autores atribuyen este incremento de las MPC a una sobreestimación del riesgo de CMC por parte de las pacientes, junto con la generalización del acceso a reconstrucción inmediata ${ }^{1,6}$.

Hasta la publicación en 2017 del modelo predictivo de riesgo absoluto $C B C R i s k^{9}$, no se disponía de una herramienta cuantitativa útil para el asesoramiento individual de riesgo de CMC en mujeres con CM unilateral esporádico. Este modelo calcula el riesgo absoluto de CMC por periodos, a través de la combinación de ocho factores de riesgo: edad al diagnóstico del primer CM, terapia antiestrogénica, antecedentes 
familiares de CM de primer grado, lesiones de moderado-alto riesgo (LMAR) previas, estatus de receptores estrogénicos, densidad mamaria al diagnóstico, tipo de tumor primario y edad al nacimiento del primer hijo. Está especialmente diseñado para mujeres con CM unilateral esporádico, al no incluir información sobre mutaciones de riesgo.

Posteriormente, se evaluó la capacidad del modelo para discriminar mujeres con alto/bajo riesgo de CMC en dos cohortes independientes ${ }^{10}$, pero los datos de seguimiento solo permitieron hacerlo con un periodo de tres a cinco años. Se concluyó que, a pesar de que existen diferencias según la prevalencia del CM, las características de la cohorte y los parámetros codificados como «desconocidos», el modelo puede ser útil para el asesoramiento individual en la práctica clínica habitual.

El objetivo de este trabajo es evaluar si el CBCRisk es capaz de identificar aquellas pacientes con mayor probabilidad de presentar LMAR o malignidad oculta en la mama contralateral y poder así seleccionar aquellas que obtendrían un mayor beneficio de la MPC.

\section{Métodos}

\section{Población a estudio}

Se incluyen mujeres con un CM unilateral esporádico sometidas a MPC, entre enero de 2017 y marzo de 2019 en una única Unidad de Mama.

Se aplicaron como criterios de exclusión:

- Edad < 18 años y > 88 años (para ajustar la muestra al modelo CBCRisk, especialmente diseñado en mujeres entre 18-88).

- Pacientes con cáncer de mama y mutaciones genéticas de alto riesgo demostradas.

- Pacientes sin cáncer de mama sometidas a mastectomía reductora de riesgo bilateral.

- Pacientes con cáncer de mama bilateral.

- Pacientes con carcinoma lobulillar in situ (CLIS).

- Pacientes con tumores de estirpe no ductal ni lobulillar.

- Pacientes sometidas a radioterapia supradiafragmática antes del primer diagnóstico de CM.

\section{Variables del estudio}

Se analizaron retrospectivamente dos grupos de variables: 1.Variables demográficas necesarias para el cálculo del CBCRisk:

- Edad al diagnóstico del primer CM.

- Terapia antiestrogénica para el tratamiento del tumor primario.

- Antecedentes de CM en familiares de primer grado.

- Lesiones de moderado-alto riesgo (LMAR) previas al diagnóstico de CM.

- Densidad mamaria al diagnóstico (según la clasificación de BI-RADS: predominio graso, patrón de densidades parcheadas, heterogéneas y extremadamente densas).
- Edad al nacimiento del primer hijo ( $<30$ años o nuliparidad, 30-39, $\geq 40$ ).

- Tipo de tumor primario (cáncer no invasivo [CDIS] puro, invasivo puro, mixto-CDIS e invasivo)

2. Variables clinicopatológicas:

Relativas al tumor:

- Tamaño tumor ( $\leq 2 \mathrm{~cm}, 2-5 \mathrm{~cm}, \geq 5 \mathrm{~cm})$.

- Multifocalidad.

- Estadio ganglionar.

- Quimioterapia adyuvante.

- Positividad receptores estrogénicos (terapia hormonal).

- Positividad HER2 + (terapia con trastuzumab).

- Hallazgos en piezas de MPC.

Se consideraron lesiones de moderado-alto riesgo (LMAR), la hiperplasia atípica (HA) ductal o lobulillar y el carcinoma lobulillar in situ (CLIS).

\section{Análisis estadístico}

Se compararon los hallazgos anatomopatológicos en las piezas de MPC con el CBCRisk a cinco años calculado con el modelo mediante la calculadora online (disponible en https:// cbc-predictor-utd.shinyapps.io/CBCRisk/). Se consideró exclusivamente el CBCRisk a los cinco años.

Se realiza en primer lugar un análisis descriptivo de las variables a estudio: frecuencias relativas y absolutas (v. cualitativas) y media y desviación estándar (DE) (v. cuantitativas). Se evalúa la relación entre variables cualitativas mediante el test de $\chi^{2}$. Para comparar medias entre dos grupos independientes, se utiliza el test $U$ de MannWhitney o $t$ de Student, según criterios de normalidad. Análisis de regresión logística univariante para identificar factores predictivos de LMAR y/o cáncer de mama contralateral oculto. Se establece el nivel de significación estadística para un valor p menor a 0,05. Para todo, el análisis ha utilizado el programa estadístico SPSS 22.0, este estudio fue aprobado por el Comité Ético del centro (Hospital Clínico Universitario Lozano Blesa, Zaragoza, España).

\section{Resultados}

\section{Descripción de la población del estudio}

Se intervinieron un total de 42 mujeres realizando mastectomía bilateral por un cáncer de mama unilateral esporádico. Las características demográficas y clínicopatológicas se describen por rangos de edad en la tabla 1.

La edad media del conjunto fue de 48,61 años (DE 10,56). Solo 13 de ellas (30,95\%), contaban con historia familiar de CM. El tipo de tumor más frecuente de la muestra en todos los grupos de edad fue el CDI (76,2\%). Los tumores primarios en 22 casos fueron multifocales $(52,38 \%)$ y asociaron LMAR hasta en 20 mujeres (47,61\%). La mayoría de los tumores fueron diagnosticados con un tamaño $\leq 2 \mathrm{~cm}(59,52 \%)$ y sin extensión ganglionar $(54,77 \%)$. 
Tabla 1 - Datos demográficos y clinicopatológicos

\begin{tabular}{|c|c|c|c|c|}
\hline & $\begin{array}{c}\text { Mujeres } \\
<40 \text { años } \\
n=6\end{array}$ & $\begin{array}{c}\text { Mujeres } \\
\text { 40-49 años } \\
\text { n = } 19\end{array}$ & $\begin{array}{c}\text { Mujeres } \\
\text { 50-59 años } \\
\text { n }=9\end{array}$ & $\begin{array}{c}\text { Mujeres } \\
>60 \text { años } \\
n=8\end{array}$ \\
\hline $\begin{array}{l}\text { Historia familiar } \\
\text { Tipo de tumor }\end{array}$ & 3 & 5 & 3 & 2 \\
\hline CDI & 6 & 13 & 7 & 6 \\
\hline CLI & 0 & 4 & 1 & 2 \\
\hline CDIS & 0 & 2 & 1 & 0 \\
\hline LMAR asociadas & 1 & 9 & 5 & 5 \\
\hline Multifocal & 3 & 11 & 3 & 5 \\
\hline $\begin{array}{l}\text { Estadio ganglionar + } \\
\text { Tamaño }\end{array}$ & 3 & 8 & 4 & 4 \\
\hline$\leq 2$ & 4 & 11 & 4 & 6 \\
\hline $2-5$ & 1 & 7 & 4 & 2 \\
\hline$\geq 5$ & 1 & 1 & 1 & 0 \\
\hline Quimioterapia adyuvante & 5 & 6 & 5 & 3 \\
\hline ER/terapia endocrina & 3 & 14 & 6 & 7 \\
\hline HER $2+$ /terapia con trastuzumab & 2 & 6 & 2 & 1 \\
\hline
\end{tabular}

CDI: carcinoma ductal infiltrante; CLI: carcinoma lobulillar infiltrante; CDIS: carcinoma ductal in situ; ER: estrogen-receptor, receptores estrogénicos; HER 2+: human epidermalgrowth factor receptor 2, receptor del factor de crecimiento epidérmico humano 2 .

El $71,42 \%$ de las mujeres fueron sometidas a terapia endocrina debido al estatus hormonal del tumor primario. Sin embargo, solo el 45,23 y el $26,19 \%$ recibieron quimioterapia y terapia con trastuzumab, respectivamente.

Riesgo calculado y hallazgos anatomopatológicos de CMC y LMAR en piezas de MPC.

El CBCRisk calculado medio a los cinco años fue de 1,87 (DE $0,91)$, es decir, el riesgo absoluto de CMC medio a cinco años en nuestra serie fue del $1,87 \%$.
Se encontraron cuatro LMAR (9,52\%), tres hiperplasias atípicas $(7,14 \%)$ y un CLIS $(2,38 \%)$ y un cáncer no invasivo (CDIS) (2,38\%). No se diagnosticó ningún carcinoma infiltrante.

El CBCRisk medio a cinco años de las pacientes con hallazgos en las piezas de MPC fue de 2,08 (DE 0,97), ligeramente superior al CBCRisk medio del conjunto $(1,87 \pm$ $0,91)$ y del subgrupo de MPC, sin hallazgos patológicos $(1,84 \pm$ 0,91). La paciente que presentó CDIS en la pieza de MC, tenía un CBCRisk calculado de 3,13 (sin ser estadísticamente

Tabla 2 - CBCRisk calculado a 5 años y hallazgos anatomopatológicos en piezas MPC

\begin{tabular}{llll} 
& CBCRisk a 5 años* & Hallazgos AP en MPC & Valor p \\
\hline Toda la serie (n 42) & 1,87 & $11,9 \%(5 / 42)$ & - \\
Pacientes sin hallazgos en MPC (n 37) & 1,84 & & \\
Pacientes con hallazgos en MPC (n 5) & 2,08 & $2,38 \%(1 / 42)$ & - \\
Hallazgo CMC & 3,13 & $9,52 \%(4 / 42)$ & 0,41 \\
Hallazgo LMAR & 1,82 & $16,67 \%(1 / 6)$ & 0,83 \\
Edad < 40 años (n 6) & 2,34 & $10,52 \%(2 / 19)$ & 0,37 \\
Edad 41-49 años (n 19) & 1,64 & $25 \%(2 / 8)$ & 0,4 \\
Edad 50-59 años (n 9) & 2,10 & $0 \%(0 / 8)$ & 0,87 \\
Edad $>60$ años (n 8) & 1,79 & & - \\
\hline
\end{tabular}

* Se expresan los valores medios.

AP: anatomía patológica.

Tabla 3 - Análisis factores predictores de CMC y/o LMAR

\begin{tabular}{|c|c|c|c|c|}
\hline Variables & MPC con hallazgos (n 5) & MPC sin hallazgos (n 37) & OR & Valor $\mathrm{p}$ \\
\hline Edad $<40$ & 1 & 5 & 1,6 & 0,70 \\
\hline Edad $>60$ & 0 & 8 & 0 & 0,25 \\
\hline Historia familiar & 2 & 11 & 1,57 & 0,64 \\
\hline Multifocalidad & 4 & 18 & 4,22 & 0,19 \\
\hline Tamaño $>=5$ & 1 & 2 & 4,38 & 0,23 \\
\hline Estado ganglionar + & 3 & 16 & 1,97 & 0,48 \\
\hline ER/terapia endocrina & 3 & 27 & 0,56 & 0,55 \\
\hline HER 2 +/terapia con trastuzumab & 2 & 9 & 2,07 & 0,45 \\
\hline
\end{tabular}


significativa esta diferencia) y su tumor primario se trataba de un CDIS T1NO con receptores hormonales positivos, no precisando quimioterapia. Los resultados se exponen en la tabla 2.

Cabe destacar que al igual que en el artículo original, las variables menos disponibles en la historia de las pacientes han sido la densidad de la mama al diagnóstico y la edad al nacimiento del primer hijo, por lo que el modelo puede ser calculado, pero requiere una interpretación cautelosa.

\section{Factores predictores de CMC y LMAR}

Se realizó un análisis univariante tratando de identificar factores predictores de CMC y/o LMAR.

Ninguna de las variables analizadas alcanzó la significación estadística, probablemente por tratarse de un tamaño muestral pequeño (tabla 3).

Se evaluaron en la muestra distintos puntos de corte del modelo CBCRisk, encontrando el CBCRisk $\geq 3$, como único valor estadísticamente significativo $(p=0,04)$, para la predicción de hallazgos patológicos en la pieza de mastectomía contralateral.

\section{Discusión}

El riesgo de CMC en la población general oscila entre 0,1-0,6\% anual $^{11}$. Sin embargo, se han descrito factores individuales y derivados del tratamiento del tumor primario que podrían modificar este riesgo.

En el estudio publicado por King et al. ${ }^{12}$, se identificó CMC oculto en $6 \%$ y LMAR en $28 \%$. En el análisis univariado, la multifocalidad/multicentricidad fue el único factor asociado con CMC (OR 2,88, p = 0,04). Sin embargo, cuando realizaron análisis multivariante, encontraron asociación entre la edad y la positividad del receptor de progesterona. Los autores concluyeron que, por el momento, y hasta que no se identificasen predictores confiables, las bajas tasas de CMC oculto no justificaban el uso de MPC en mujeres con riesgo medio.

La incidencia de hallazgos patológicos en nuestra serie fue menor (9,52\% para LMAR y 2,38\% para CO). No se diagnosticó enfermedad invasiva, lo que hace todavía más difícil valorar el verdadero beneficio de la MPC para la mayoría de las mujeres.

El modelo del CBCRisk recoge factores de riesgo previamente reconocidos en la literatura. Sin embargo, se rechazaron para su diseño otros factores también bien conocidos. La menor edad al diagnóstico, presencia de antecedentes familiares de primer grado, los receptores estrogénicos negativos y el nacimiento del primer hijo, más allá de los 40 años, se asociaron con mayor incidencia de CMC. Se descartaron el tamaño tumoral y número de ganglios afectados, siendo que previamente se había considerado que un tamaño tumoral entre 2 y $5 \mathrm{~cm}$ incrementaría el riesgo relativo (RR) 1,51 y tumores $>5 \mathrm{~cm}$ presentarían un $\mathrm{RR}$ del 1,89 , así como que la afectación de más de 10 ganglios obtendría un $\mathrm{RR} 1,62^{13}$. Se desestimó la valoración de antecedentes familiares diferentes al primer grado, cuando es conocido que la influencia de la historia familiar responde a un sistema complejo, pero el riesgo parece superior en caso de antecedentes múltiples, o si además de un familiar de primer grado se asocia un familiar de segundo grado ${ }^{13}$. También se rechazaron la presencia de menopausia, la positividad para HER-2, historia personal o familiar de cáncer de ovario, terapias de reemplazo hormonal e índice de masa corporal.

Por otra parte, se desconoce si la combinación de determinados factores puede aumentar exponencialmente el riesgo. Otra limitación del modelo es que no se diferencia entre el tipo de terapia antiestrogénica administrada, habiéndose demostrado ya que la reducción de riesgo de CMC es diferente, en caso de administrar tamoxifeno e inhibidores de la aromatasa (50 vs. 70\%, respectivamente, de reducción de riesgo en no portadoras) $)^{14}$.

La calculadora permite, excepto para la edad al diagnóstico y características del tumor primario, la opción «desconocido» en todas las categorías. Lo cual tiene la ventaja de poder calcular el CBCRisk, aun con escasa información, pero asumiendo su valor limitado. No se ha establecido el número máximo de variables que pueden codificarse como "desconocido", sin que el CBCRisk pierda completamente su valor. Este problema se resolvería en caso de recogida prospectiva de los datos.

Por otra parte, no se han determinado los valores de CBCRisk, a partir de los cuales podría recomendarse la MPC en la población general. Los valores considerados como «riesgo bajo» difirieron en las dos cohortes de validación $\left(2,4\right.$ y 1,53) ${ }^{10}$, ninguno de estos límites ha resultado estadísticamente significativo en nuestra muestra $(p=0,37$ y $p=0,64$, respectivamente). Si consideramos como hemos mencionado anteriormente que el riesgo anual máximo en la población general es de 0,6\%, podríamos considerar como límite el CBCRisk a cinco años $\geq 3$. En nuestra muestra, el 11,9\% de las pacientes tienen un CBCRisk $\geq 3$, encontrándose entre ellas el $40 \%(2 / 5)$ de los hallazgos patológicos y el único CDIS diagnosticado. Además, en nuestro conjunto de pacientes, el CBCRisk $\geq 3$, este es el único valor de corte que ha alcanzado la significación estadística ( $p=0,04)$.

Este estudio cuenta con limitaciones metodológicas: una muestra pequeña y recogida de datos retrospectiva (motivo por el que no es infrecuente la elección de «desconocido» para el cálculo del modelo). Sin embargo, consideramos que el CBCRisk puede resultar útil en la práctica clínica habitual, ya que hemos observado una pequeña diferencia entre los valores de CBCRisk a cinco años de las pacientes con hallazgos patológicos en la pieza de MPC y el valor CBCRisk $\geq 3$ ha resultado estadísticamente significativo en nuestra muestra. Se precisan estudios con mayor tamaño muestral y diseño prospectivo.

En conclusión, urge el desarrollo de herramientas cuantitativas para el asesoramiento individual del riesgo de CMC en mujeres con CM unilateral esporádico, que permitan evitar las MPC innecesarias. Las pacientes deben ser adecuadamente informadas de los riesgos y beneficios esperables de la MPC, en cada caso particular.

\section{Financiación}

Este trabajo no ha recibido ningún tipo de financiación. 


\section{Conflicto de intereses}

Los autores declaran no tener ningún conflicto de intereses.

B I B L I O G R A F Í A

1. Wright FC, Look Hong NJ, Quan ML, Beyfuss K, Temple S, Covelli A, et al. Indications for contralateral prophylactic mastectomy: A consensus statement using modified delphi methodology. Ann Surg. 2018;267:271-9.

2. Osman F, Saleh F, Jackson TD, Corrigan MA, Cil T. Increased postoperative complications in bilateral mastectomy patients compared to unilateral mastectomy: an analysis of the NSQIP database. Ann Surg Oncol. 2013;20:3212-7.

3. Alves Nogueira Fabro E, Bergmann A, do Amaral E. Silva B, Padula Ribeiro AC, de Souza Abrahão kE, da Costa Leite Ferreira MG, et al. Post-mastectomy pain syndrome: incidence and risks. Breast. 2012;21:321-5.

4. Frost MH, Hoskin TL, Hartmann LC, Degnim AC, Johnson JL, Boughey JC. Contralateral prophylactic mastectomy: longterm consistency of satisfaction and adverse effects and the significance of informed decision-making, quality of life, and personality traits. Ann Surg Oncol. 2011;18:3110-6.

5. Bedrosian I, Hu CY, Chang GJ. Population-based study of contralateral prophylactic mastectomy and survival outcomes of breast cancer patients. J Natl Cancer Inst. 2010;102:401-9.

6. Tuttle TM, Habermann EB, Grund EH, Morris TJ, Virnig BA. Increasing use of contralateral prophylactic mastectomy for breast cancer patients: a trend toward more aggressive surgical treatment. J Clin Oncol. 2007;25:5203-9.

7. Güth U, Myrick ME, Viehl CT, Weber WP, Lardi AM, Schmid $\mathrm{SM}$. Increasing rates of contralateral prophylactic mastectomy - a trend made in USA? Eur J Surg Oncol. 2012;38:296-301.

8. Early Breast Cancer Trialists' Collaborative Group (EBCTCG). Effects of chemotherapy and hormonal therapy for early breast cancer on recurrence and 15-year survival: an overview of the randomised trials. Lancet. 2005;365:1687717 .

9. Chowdhury M, Euhus D, Onega T, Biswas S, Choudhary PK. A Model for Individualized Risk Prediction of Contralateral Breast Cancer. Breast Cancer Res Treat. 2017;161:153-60.

10. Chowdhury M, Euhus D, Arun B, Umbricht C, Biswas S, Choudhary P. Validation of a personalized risk prediction model for contralateral breast cancer. Breast Cancer Res Treat. 2018;170:415-23.

11. Boughey JC, Attai DJ, Chen SL, Cody HS, Dietz JR, Feldman $\mathrm{SM}$, et al. Contralateral Prophylactic Mastectomy (CPM) Consensus Statement from the American Society of Breast Surgeons: Data on CPM Outcomes and Risks. Ann Surg Oncol. 2016 Oct;23:3100-5.

12. King TA, Gurevich I, Sakr R, Patil S, Stempel M, Morrow M. Occult Malignancy in Patients Undergoing Contralateral Prophylactic Mastectomy. Ann Surg. 2011;254:2-7.

13. Vichapat V, Gillett C, Fentiman IS, Tutt A, Holmberg L, Lüchtenborg M. Risk factors for metachronous contralateral breast cancer suggest two aetiological pathways. Eur J Cancer. 2011;47:1919-27.

14. Basu NN, Barr L, Ross GL, Evans DG. Contralateral RiskReducing Mastectomy: Review of Risk Factors and RiskReducing Strategies. Int J Surg Oncol. 2015;2015:901046. 